\title{
Computer implementation of the international standards for neurological classification of spinal cord injury for consistent and efficient derivation of its subscores including handling of data from not testable segments
}

\author{
Schuld, C ; Wiese, J ; Hug, A ; Putz, C ; van Hedel, H J A ; Spiess, M R ; Weidner, N
}

\begin{abstract}
The International Standards for Neurological Classification of Spinal Cord Injury (ISNCSCI), defined by the American Spinal Injury Association (ASIA), and particularly the ASIA Impairment Scale (AIS) are widely used for research and clinical purposes. Although detailed procedures for scaling, scoring, and classification have been defined, misclassifications remain a major problem, especially for cases with missing (i.e., not testable $[\mathrm{NT}]$ ) data. This work aimed to implement computer-based classification algorithms that included rules for handling NT data. A consistent and structured algorithmic scoring, scaling, and classification scheme, and a computerized application have been developed by redefining logical/mathematical imprecisions. Existing scoring rules are extended for handling NT segments. Design criterion is a pure logical approach so that substitution of non-testability for all valid examination scores leads to concordant results. Nine percent of 5542 datasets from 1594 patients in the database of the European Multicenter Study of Human Spinal Cord Injury (EM-SCI) contained NT segments. After adjusting computational algorithms, the classification accuracy was equivalent between clinical experts and the computational approach and resulted in $84 \%$ valid AIS classifications within datasets containing NT. Additionally, the computational method is much more efficient, processing approximately 200,000 classifications/sec. Computational algorithms offer the ability to classify ISNCSCI subscores efficiently and without the risk of human-induced errors. This is of particular clinical relevance, since these scores are used for early predictions of neurological recovery and functional outcome for patients with spinal cord injuries.
\end{abstract}

DOI: https://doi.org/10.1089/neu.2011.2085

Posted at the Zurich Open Repository and Archive, University of Zurich

ZORA URL: https://doi.org/10.5167/uzh-55371

Journal Article

Published Version

Originally published at:

Schuld, C; Wiese, J; Hug, A; Putz, C; van Hedel, H J A; Spiess, M R; Weidner, N (2012). Computer implementation of the international standards for neurological classification of spinal cord injury for consistent and efficient derivation of its subscores including handling of data from not testable segments. Journal of Neurotrauma, 29(3):453-461.

DOI: https://doi.org/10.1089/neu.2011.2085 


\title{
Computer Implementation of the International Standards for Neurological Classification of Spinal Cord Injury for Consistent and Efficient Derivation of Its Subscores Including Handling of Data from Not Testable Segments
}

\author{
Christian Schuld, Julia Wiese,, Andreas Hug,, Cornelia Putz, \\ Hubertus J.A. van Hedel, ${ }^{3}$ Martina R. Spiess, ${ }^{3}$ Norbert Weidner, \\ EM-SCl Study Group and Rüdiger Rupp ${ }^{1}$
}

\begin{abstract}
The International Standards for Neurological Classification of Spinal Cord Injury (ISNCSCI), defined by the American Spinal Injury Association (ASIA), and particularly the ASIA Impairment Scale (AIS) are widely used for research and clinical purposes. Although detailed procedures for scaling, scoring, and classification have been defined, misclassifications remain a major problem, especially for cases with missing (i.e., not testable [NT]) data. This work aimed to implement computer-based classification algorithms that included rules for handling NT data. A consistent and structured algorithmic scoring, scaling, and classification scheme, and a computerized application have been developed by redefining logical/mathematical imprecisions. Existing scoring rules are extended for handling NT segments. Design criterion is a pure logical approach so that substitution of nontestability for all valid examination scores leads to concordant results. Nine percent of 5542 datasets from 1594 patients in the database of the European Multicenter Study of Human Spinal Cord Injury (EM-SCI) contained NT segments. After adjusting computational algorithms, the classification accuracy was equivalent between clinical experts and the computational approach and resulted in $84 \%$ valid AIS classifications within datasets containing NT. Additionally, the computational method is much more efficient, processing approximately 200,000 classifications/sec. Computational algorithms offer the ability to classify ISNCSCI subscores efficiently and without the risk of human-induced errors. This is of particular clinical relevance, since these scores are used for early predictions of neurological recovery and functional outcome for patients with spinal cord injuries.
\end{abstract}

Keywords: algorithm; ASIA Impairment Scale; computer aided classification; reliability; sacral sparing

\section{Introduction}

T He International Standards for Neurological Classification of Spinal Cord Injury (ISNCSCI) (Marino et al., 2003; Furlan et al., 2011; American Spinal Injury Association, 2003), defined by the American Spinal Injury Association (ASIA), are commonly used to quantify neurological deficits and predict functional outcome in patients with acute spinal cord injury (SCI). Based on the examination of motor and sensory functions, a set of derived/output variables are defined: (1) motor and sensory scores; (2) neurological levels; (3) a completeness criterion; (4) zones of partial preservation; and (5) the ASIA Impairment Scale (AIS). These derived variables are clinically meaningful, for instance, as early predictors of recovery (van Middendorp et al., 2011), or for the purpose of sub-grouping/stratification and as outcome measures in clinical studies (Ditunno, 2010; Fawcett et al., 2007).

Reliability issues have been raised with respect to both the clinical testing and the derivation of scores (Cohen et al., 1998). Testing reliability is improved by training (Mulcahey et al., 2007), and frequent examinations of heterogeneous patient collectives. Scoring reliability is improved by formal training (Chafetz et al., 2008), and analysis of meaningful

\footnotetext{
${ }^{1}$ Heidelberg University Hospital, Spinal Cord Injury Center, Heidelberg, Germany.

${ }^{2}$ Heidelberg University Hospital, Department of Orthopedic and Trauma Surgery, Heidelberg, Germany.

${ }^{3}$ Balgrist University Hospital, Spinal Cord Injury Center, Zurich, Switzerland.
} 
numbers of ISNCSCI datasets on a regular basis. Nevertheless, misclassification rates ranging from 9-11\% (Chafetz et al., 2008, Rupp et al., unpublished data from the EM-SCI project) signify a need for further improvement in the framework of clinical trials.

Accurate classifications (with a focus on the AIS) are based on several rules and conventions (e.g., prediction of motor function from sensory function). Moreover, ISNCSCI does not cover all borderline cases (e.g., low sacral region). Thus, the aim of the present study is to reveal open issues of the ISNCSCI by developing comprehensive algorithms for both the manual and computational scoring of all derived ISNCSCI variables. These algorithms compose not only the basis for more reliable manual scaling, scoring, and classification, but also a prerequisite for computer applications. The enormous advantages of validated computerized ISNCSCI implementation are (1) the elimination of sources of human error, and (2) a tremendous reduction in the time required for analyzing large datasets.

Computational SCI-scoring systems have been established, but unlike Linassi's (Linassi et al., 2010) and Chafetz's (Chafetz et al., 2009) ISNCSCI computer programs, the presented algorithms additionally cover the problem of segments that are not testable (NT). The frequency of NT components (1-10 segments) per dataset in the European Multicenter Study of Human Spinal Cord Injury (EM-SCI) database (February 2009 ) is almost $9 \%$. Despite their clinical value, these datasets are most likely discarded due to difficulties with manual scoring. Therefore, the aim of the present study was to develop and validate new algorithms which implement rules for processing incomplete datasets.

\section{Methods}

The present work originates from the EM-SCI study (Curt et al., 2004). For the design of the computational algorithms, a first prerequisite is the formal notation of all input and output variables, including the domains of definition.

\section{Input variables (source data)}

The neurological assessment consists of a motor examination, two sensory modalities (pinprick discrimination and light touch appreciation) and an anorectal examination. Pinprick (PP) discrimination and light touch (LT) appreciation is tested in 28 dermatomes on the right and left sides of the body. Grading is done on a three-point scale ( 0 , absent, 1 , impaired, 2, normal). Segments that cannot be accurately tested (e.g., due to casts or burns) are recorded as NT. The mathematical denotations are presented in Supplemental Table 1 (see online supplementary material at http://www.liebertonline.com).

Motor scores (MS) are assessed in 10 segmental myotomes for the right and left body sides. These myotomes are equally distributed in the upper and lower extremity. Grading is done on a six-point manual muscle-testing scale that varies from 0 (no visible or palpable muscle contraction) to 5 (normal function). Segments that cannot be accurately tested are recorded as NT. For the full definitions, see the reference manual (American Spinal Injury Association, 2003). Mathematical denotations are presented in Supplemental Table 1 (see online supplementary material at http://www.liebertonline.com). The anorectal examination tests for presence of any (deep) anal sensation (AAS) and voluntary anal contraction (VAC).
Both variables are graded as absent (0) or present (1). Inconclusive examinations are recorded as NT (see also Supplemental Table 1; see online supplementary material at http:// www.liebertonline.com). Overall, these exams result in 134 input variables.

\section{Output variables (derived data)}

For PP, LT, and MS, the following sum scores are defined: upper extremity motor score (UEMS), lower extremity motor score (LEMS), total motor score (TMS), right/left pinprick score (LPP and RPP), and right/left light touch score (LLT, RLT; see also Supplemental Table 1; see online supplementary material at http://www.liebertonline.com).

Determinants for SCI levels are the following variables: right/left sensory level (RSL and LSL), right/left motor level (RML and LML), and a single neurological level of injury (NLI), defined as the most caudal intact segment with intact motor and sensory function below which deficits exist. All levels can vary between C1 and S4-S5. A level is graded as intact if all segments in the source are graded as intact (see also Supplemental Table 1; see online supplementary material at http://www.liebertonline.com).

SCI severity is described by the variables AIS and "completeness" (COMP; Supplemental Table 1; see online supplementary material at http://www.liebertonline.com). The AIS variable consists of a five-point scale (A, complete; $\mathrm{B}$, sensory incomplete, motor complete; $\mathrm{C}$, sensory and motor incomplete; D, sensory and motor incomplete, and at least half of the key muscles below the NLI have a grade $\geq 3$; E, normal; see Table 1 for the full definition). COMP is denoted as incomplete or complete.

Zones of partial preservation are defined only for complete injuries and describe preserved function below the level of injury: right/left sensory zones of partial preservation (RSZPP and LSZPP), and right/left motor zones of partial

Table 1. Definition of the ASIA Impairment Scale (AIS)

\begin{tabular}{ll}
$\begin{array}{l}\text { ASIA } \\
\text { Impairment }\end{array}$ & \multicolumn{1}{c}{ Description } \\
\hline A & $\begin{array}{c}\text { Complete. No sensory or motor function } \\
\text { is preserved in the sacral segments S4-S5. } \\
\text { Incomplete. Sensory but not motor function } \\
\text { is preserved below the neurological level } \\
\text { and includes the sacral segments S4-S5. } \\
\text { Incomplete. Motor function is preserved below } \\
\text { the neurological level, and more than half } \\
\text { of key muscles below the neurological level } \\
\text { have a muscle grade less than 3 (grades 0-2). } \\
\text { Incomplete. Motor function is preserved below } \\
\text { the neurological level, and at least half } \\
\text { of key muscles below the neurological } \\
\text { level have a muscle grade greater than } \\
\text { or equal to 3. }\end{array}$ \\
N $\quad \begin{array}{l}\text { Normal. Sensory and motor function } \\
\text { are normal. }\end{array}$
\end{tabular}

For an individual to receive a grade of $\mathrm{C}$ or $\mathrm{D}$, he/she must be incomplete, that is, have sensory or motor function in the sacral segments S4-S5. In addition, the individual must have either (1) voluntary anal sphincter contraction, or (2) sparing of motor function more than three levels below the motor level. 
preservation (RMZPP and LMZPP). Overall, these variables account for 18 output arguments.

\section{Algorithms for computational scoring, scaling, and classification}

Based on the formalization process, algorithms have been developed using the ANSI C (ISO/IEC 9899:1999) programming language, as well as Microsoft Visual $\mathrm{C}++$ (Version 13.10.3077) and GNU (version 3.4.4 cygming special, gdc 0.12, using dmd 0.125) compilers. ANSI $C$ was chosen because it is the most commonly used programming language worldwide (Bieman and Murdock, 2001). This enables a wide range of applications, including maximum portability across different computer platforms (tested: win32, linux i386/amd64, and aix), and easy integration into various other projects.

Algorithms will be described for both manual and computational calculation of all output variables mentioned above, starting with the simple formulas (the sum scores) and ending up with the most complicated determination (the AIS, with consideration of NT segments). To be able to use normal arithmetic and logical operations on spinal segments, all variables containing segmental data are encoded as follows: $\{\mathrm{C} 1=0, \mathrm{C} 2=1, \ldots, \mathrm{S} 4-\mathrm{S} 5=28, \mathrm{Coccyx} 1(\mathrm{Co} 1)=29\}$.

\section{Handling of NT segmental assessment data}

The term "determinability" is introduced for each output variable to check if a variable is uniquely computable despite NT segments in the source data.

The ISNCSCI contains general rules for determinability of sensory scores and motor scores only. For all other output variables, dedicated rules are presented in this article. The single mandatory requirement for these rules is the unique computability of any given output variable. For a positive determinability, NT substitution for all possible grades must lead to concordant classifications. Accordingly, discordant classifications make the output variable undeterminable and thus not available (NA).

\section{Calculation of sum scores}

Sum scores (UEMS, LEMS, TMS, RLT, LLT, RPP, and LPP) are determinable if all summands in the source data are valid (i.e., not NT). The existence of at least one NT summand leads to an undeterminable sum score.

\section{Determination of sensory levels}

The SCI level variables (sensory / motor levels and sensory / motor ZPPs) are defined equally for each side, therefore we describe them for one side only. The algorithm for the sensory levels is defined as follows: Search for the first appearance of a grade unequal to 2 in LT or PP on a given side, starting at C2 and moving caudally segment-wise. The last normal segment, rostral to the identified dermatome, is the sensory level. If the sensation of the C2 dermatome is already impaired, the sensory level is recorded as $\mathrm{C} 1$ based on the definition of the latest ISNCSCI revision (ASIA, 2011).

In case of NT data in LT or PP, determinability must be checked separately. (1) NT data below the sensory level do not influence the sensory levels. (2) Sensory level is applicable despite the presence of NT data if either LT or PP of a more rostral segment is graded 0 or 1 , since a grade of 0 or 1 is
Table 2. Determinability Sensory Levels, Determinability Sensory ZPPS, and Transfer Function: Virtual Muscle Grades for Not Assessable Mytomes

\begin{tabular}{lcccc}
\hline $\begin{array}{l}\text { LT } \\
\text { grade }\end{array}$ & $\begin{array}{c}\text { PP } \\
\text { grade }\end{array}$ & $\begin{array}{c}\text { Transfer } \\
\text { function virtual } \\
\text { muscle grades }\end{array}$ & $\begin{array}{c}\text { Determinability } \\
\text { sensory levels }\end{array}$ & $\begin{array}{c}\text { Determinability } \\
\text { sensory ZPPs }\end{array}$ \\
\hline 2 & 2 & 5 & Yes & Yes \\
2 & 1 & 1 & Yes & Yes \\
2 & 0 & 1 & Yes & Yes \\
2 & NT & NT & No & Yes \\
1 & 2 & 1 & Yes & Yes \\
1 & 1 & 1 & Yes & Yes \\
1 & 0 & 1 & Yes & Yes \\
1 & NT & 1 & Yes & Yes \\
0 & 2 & 1 & Yes & Yes \\
0 & 1 & 1 & Yes & Yes \\
0 & 0 & 0 & Yes & Yes \\
0 & NT & $0 / 1$ & Yes & No \\
NT & 2 & NT & No & Yes \\
NT & 1 & 1 & Yes & Yes \\
NT & 0 & $0 / 1$ & Yes & No \\
NT & NT & NT & No & No \\
\hline
\end{tabular}

For each of the $16 \mathrm{LT} / \mathrm{PP}$ combinations the determinabilities and the resulting virtual motor grade are shown.

NT, not testable; LPP, left pinprick score; LT, light touch score; PP, pinprick score; ZPP, zone of partial preservation.

sufficient to pinpoint the sensory level to this segment. (3) For all other cases, see Table 2, column "Determinability sensory levels," which provides a full listing of all LT/PP combinations and their determinability for sensory levels. Please see also Supplemental Table 2 (see online supplementary material at http://www.liebertonline.com) for the logical expressions.

\section{Determination of motor levels}

Unlike the dermatomes for the sensory level determination, myotomes are not defined for the following segments: C2-C4, T2-L1, and S2-S5. To overcome this limitation, the standards stipulate the use of sensory tests as proxies for motor tests. Yet, the term "If sensation is impaired, motor function is considered impaired" (American Spinal Injury Association, 2003, p. 52) is imprecise, since it does not describe how much impairment is assumed in motor function. This information is nonetheless important for the determination of the motor level. A segment graded 3 or 4 is considered to be normal, if the next rostral level is graded 5. Therefore, it is important whether the sensory impairment is converted to a motor score grade of $<3$ or $\geq 3$. Recently, a clarification of this topic was published (Waring et al., 2010). Impairment in LT or PP is translated into a virtual muscle grade of 1 . Still, rules for handling NT segments are still missing. A precise dedicated transfer function resolving all 16 possible combinations of LT and PP grades for a given segment lacking a key muscle definition is shown in Table 2, column "transfer function virtual muscle grade." Please see also Supplemental Table 2 (see online supplementary material at http://www.liebertonline.com) for the logical expression. This transfer function enables the calculation of so-called virtual muscle grades of the regions C2-C4, T2-L1, and S2-S5 for further processing.

The variability in motor level calculation, depending on the interpretation of this rule, applies to only one segment. Using 
a grade of $<3$ leads to a motor level that is one segment more rostral than using a grade of $\geq 3$. The most recent ASIA statement confirms this decision: "If either light touch or pinprick are abnormal, then this is equivalent to the C4 (L1) motor being grade 1 . The motor level definition can be applied using the derived C4 (L1) grades" (Waring et al., 2010).

The advantage of applying the transfer function to calculate virtual muscle grades for NT segments is that the MS variable can be processed like the LT and PP variables. The algorithm for motor level determination is similar to the sensory level algorithm, except that a myotome graded 3 or 4 is considered to be intact, given that the next rostral myotome is tested as normal (5). The ML algorithm is defined as follows: Use the transfer function to fill in virtual scores for segments C2-C4, T2-L1, and S2-S5, and search for the first muscle grade unequal to 5 starting with $\mathrm{C} 2$ proceeding caudally segmentwise. The segment rostrally to the one identified is considered the motor level, except if that specific muscle grade is 3 or 4 . In that case, the motor level is caudally adjusted by exactly one segment.

\section{Zones of partial preservation}

ZPPs are applicable in complete lesions only and refer to those segments caudal to the sensory and motor levels which remain partially innervated (American Spinal Injury Association, 2003, p. 53-54).

The standards do not state whether motor ZPPs follow sensory ZPPs for myotomes without key muscle definition. Algorithms for both cases have been implemented, but the EM-SCI study uses the rule "motor ZPPs do not follow sensory ZPPs."

\section{Sensory ZPPs}

The first step is to search for the first occurrence of a segment graded unequal to 0 in LT and PP on a given side, starting at S4-S5 and moving rostrally segment-wise. This segment is defined as $\mathrm{ZPP}$, except if it is equal to the sensory level on the given side. In this case, there is by definition no zone of partial preservation. Determinability is described in detail in Table 2, column "Determinability sensory ZPPs" and Supplemental Table 2 (see online supplementary material at http://www.liebertonline.com). At least one of the sensory modalities of the segment containing one NT item must not be graded 0 , because a grade of 1 or 2 in one modality and side is sufficient to pinpoint the sensory ZPP to this segment.

\section{Motor ZPPS}

Like the algorithm for motor level determination, the implicit problem of the motor ZPP algorithm is the lack of motor scores in certain segments. Therefore, a function for the calculation of virtual motor grades for not assessable myotomes, according to the sensory scores, needs to be defined. The actual implementation is user-configurable.

For the rule "MZPPs follow SZPPs," the same transfer function is used as for motor level determination. For the rule "MZPPs do not follow SZPPs," the NT myotomes are set to a virtual muscle grade of 0 .

The resulting padded MS variable is used for further processing. Search for the first segment graded unequal to 0 starting at segment S4-S5 and moving rostrally segment-wise. This segment is denoted as the motor ZPP.

\section{Single neurological level of injury}

The calculation of the NLI is straightforward if all sensory and motor levels are defined. In this case, it is the most rostral value of all four levels (Supplemental Table 2; see online supplementary material at http://www.liebertonline.com). Determinability is given by the determinabilities of the depending variables. If any of the variables RSL, LSL, RML, or LML are not determinable because of NT data, the NLI is also not determinable. However, given the fact that the NLI is a crucial input variable for the distinction between AIS C versus AIS D (see the section on key muscle counting criteria, below), a more sophisticated NLI algorithm considering NT data is necessary in order to differentiate between AIS C and D in all cases. The proposed algorithm (Fig. 1) is designed to output a list of theoretical NLIs, taking into account any NT data in MS, $\mathrm{PP}$, and LT inputs. Starting at C2 going segment-wise caudally every segment is checked for normal function and for NTs in MS, PP, and LT. For segments containing NT data all possible NLIs are recorded. A not intact but testable segment stops the algorithm. For a more detailed description see the legend of Figure 1.

\section{ASIA Impairment Scale}

The determination of the AIS (Table 1) is by far the most complicated output variable, because it compiles almost all information of the sensory and motor examination into one single parameter (Spiess et al., 2009). A decision tree (Yuan and Shaw, 1995) was used to fractionize this classification process (Fig. 2). The tree starts with the simplest decision by checking for AIS E and ends with the most difficult distinction between AIS C and AIS D. Exactly one AIS grade is excluded for each decision node by using a process of elimination. Each decision node will be described in detail in terms of decision rule, determinability, and classes of AIS grades among which to distinguish.

Normal function (decision: AIS E versus AIS A, B, C, and D) is defined as MS, PP, LT, AAS, and VAC all being graded entirely as intact. Algorithmically, this is affirmed by counting intact graded variables. If all 134 are intact, the dataset is graded as AIS E. For testing determinability, the number of NT variables is counted. If the number of intact graded variables plus the number of NT-graded variables equals 134 (the overall number of variables), the AIS grade cannot be determined, because the NT variables are decisive for the AIS grade (Supplemental Table 2; see online supplementary material at http://www .liebertonline.com).

Sacral sparing (decision AIS A versus AIS B, C, and D) is defined as "any preserved function in segment S4-S5." This includes motor function, as assessed by the VAC variable, as well as sensory function, represented by the variables AAS, LT (S4-S5, right/left), and PP (S4-S5, right/left). Absence of sacral sparing is equivalent to a complete lesion, which is checked in the "Sacral sparing?" decision tree node (Fig. 2). A complete lesion must meet the following conditions: $\mathrm{AAS}=$ absent and $\mathrm{VAC}=$ absent and LT $(\mathrm{S} 4-\mathrm{S} 5$, right $)=0$ and LT (S4-S5, left) =0 and PP (S4-S5, right) =0 and PP (S4-S5, left $)=0$. In case of NT S4-S5 variables, the AIS can still be determined if one of these six variables is graded at least as impaired or normal (Supplemental Table 2; see online supplementary material at http://www.liebertonline.com). In 


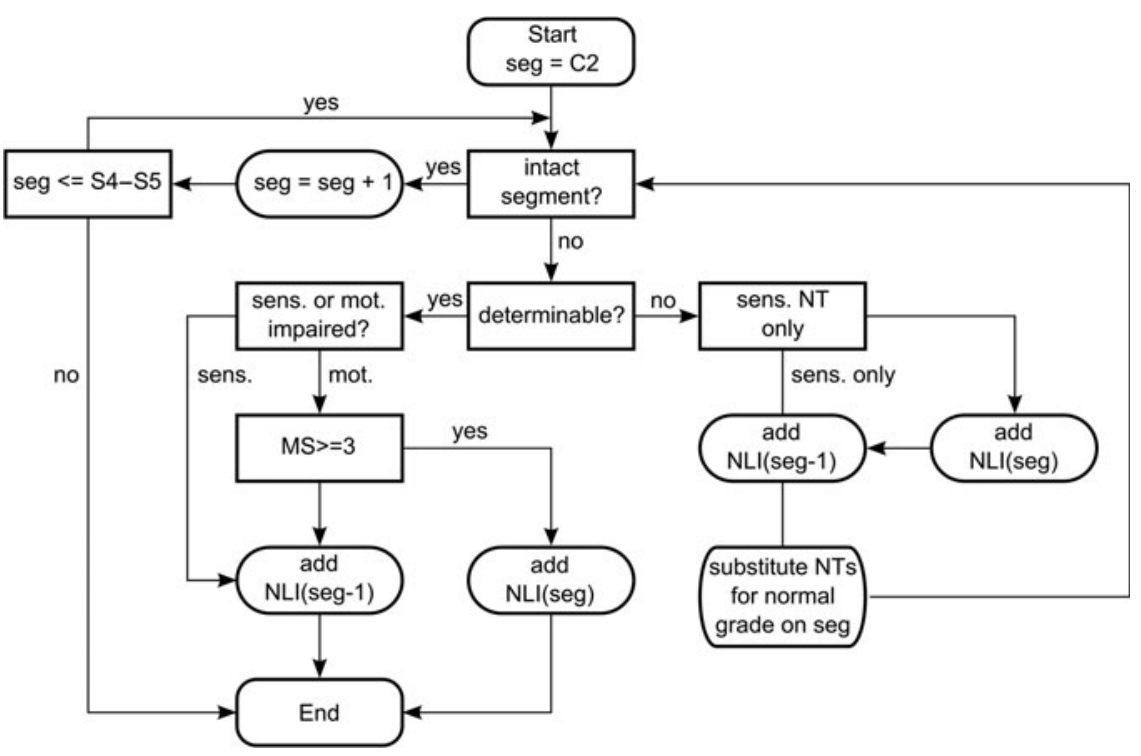

FIG. 1. Starting at segment $\mathrm{C} 2$ (seg $=\mathrm{C} 2)$, the algorithm verifies whether the segment is graded as intact in all modalities ("intact segment" in flowchart, logical expression in Supplemental Table 2; see online supplementary material at http:// www.liebertonline.com). If the segment is intact, the $\langle\operatorname{seg}\rangle$ variable is increased so that the next segment can be checked for intactness until the most caudal segment S4-S5 is reached. There are two possible causes for a non-intact segment: Either the segment is graded as not intact (left branch in the flowchart), or there are not testable (NT) data for the segment (right branch in the flowchart), which leads to an undeterminable neurological level of injury (NLI; see Supplemental Table 2 for branching condition). Variables assigned as NT are treated as if they were intact, and potential NLIs are determined. For NT data in sensory variables only, the potential NLI is $<\operatorname{seg}>-1$. NT data in the motor variables lead to two potential NLIs: <seg $>$ and $<$ seg $>-1$. After recording the NLIs, the intact grades are substituted for NT data, and the condition for intact segments is checked again for the current segment. The first step in the "determinable" impairment branch is to verify if sensory and/or motor function is impaired. For sensory impairment, the potential NLI is set to $<\mathrm{seg}>-1$. Motor impairments need an additional check for grades $\geq 3$. If true, the potential NLI is set to $\langle s e g\rangle$, because a grade of $3+$ is considered to be intact; otherwise, the potential NLI is set to $<\operatorname{seg}>-1$. The algorithm stops once impaired segments are found, because NT data below the impairment have no influence on the NLI (NLI, neurological level of injury; MS, motor score; sens., sensory; mot., motor).

other words, a grade of 1 for any of these variables is sufficient to identify an incomplete lesion.

Motor incompleteness (decision AIS B versus AIS C and D) is characterized by the presence of VAC or sparing of motor function more than three levels below the motor level. Although ZPPs are not calculated for incomplete lesions (as described above), identically calculated variables have been used as input for determining whether motor function was present more than three levels below the motor level (RMPRES and LMPRES). In case of NT data in motor scores, a more detailed approach is necessary for determinability, because sparing depends on the variables "motor level" and "motor preservation." Both variables are susceptible to NT data. To overcome this situation, the terms/variables "pessimistic" and "optimistic" motor levels and motor preservation have been introduced. When the adjective "optimistic" can describe the view of the patient for a given function, normal innervations are assumed for all NT data. For accounts labeled as "pessimistic," on the other hand, no function is assumed for all NT data. When calculating optimistic motor levels, muscle grades of 5 replaced NT inputs. For pessimistic motor levels, muscle grades of 0 replaced NT inputs (Fig. 3). The results are stored into temporary variables MS_opt and MS_pes. Motor levels and motor preservations are determined using these variables, so that an estimation of the minimal and the maximal sparing of motor function can be calculated. If minimal sparing is less than or equal to 3 , while maximal sparing is greater than 3 , the AIS is not determinable. See Figure 3 and Supplemental Table 2 (see online supplementary material at http://www.liebertonline.com) for the logical expressions.

Key muscle counting criteria (decision AIS C versus AIS D). For a grade of AIS D, at least half of the key muscles below the NLI must have a muscle grade greater than or equal to 3 . In terms of determinability, this is the most challenging rule of the AIS classification scheme, because all NT scores in LT, PP, or MS are potentially decisive for the NLI (see above). Besides that, NT data must be considered in the counting process of key muscles, as well. Hence, the overall complexity of the AIS $C$ versus $D$ decision is much higher than of all other AIS decisions. Therefore, the decision algorithm is split into two levels: The inner level consists of an algorithm based on a given NLI, which counts the overall number of key muscles (KM), muscle grades $3+(\mathrm{GT} 3)$, and instances of NT data in key muscles (iNT) in segments below the NLI. The criterion for AIS D is GT3 $\geq K M / 2$. Determinability is checked by the condition $\mathrm{GT} 3+\mathrm{iNT} \geq \mathrm{KM} / 2$. If this condition holds true while $\mathrm{GT} 3<\mathrm{KM} / 2$, the AIS $\mathrm{C}$ versus D decision-making process is aborted with "AIS not available" as the result. This is due to the fact that an NT in the motor score has been identified to be critical for distinguishing between AIS C and D. The AIS grade resulting from this inner-level process is only valid under one condition, namely the presence of a uniquely determinable NLI. In case of undeterminable NLIs 


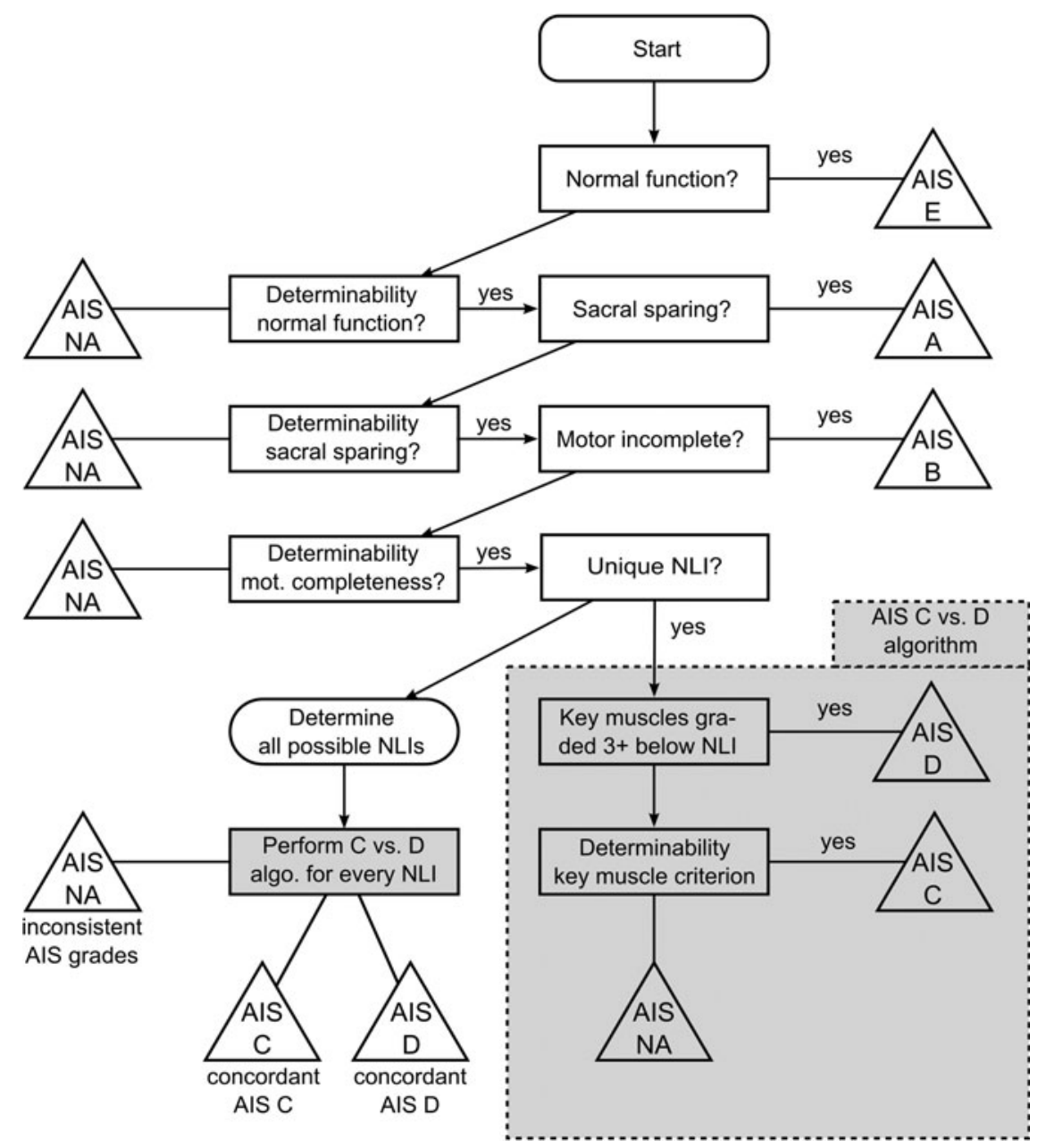

FIG. 2. Decision tree of the American Spinal Injury Association (ASIA) Impairment Scale classification process. Exactly one AIS grade (triangle) gets discarded for each decision node (rectangle) by using a process of elimination. The lower right part (grey box) contains the AIS C versus D decision-making process. This part is emphasized as it is used in the "Perform C versus D algorithm for every NLI" node (mot., motor; NLI, neurological level of injury).

caused by NT data, a list of all potential NLIs is retrieved in an outer-level decision process. AIS grades for each potential NLI are determined according to the inner-level algorithm mentioned above. Concordant AIS grades lead to a defined AIS grade despite NT data in the input variables.

\section{Completeness}

Completeness is defined as complete for AIS A lesions and as incomplete for AIS B, C, and D lesions. Completeness is not applicable for AIS E lesions.

\section{Results}

A sample of the EM-SCI database (Rupp et al., 2005) (queried in February 2009) containing 5542 ISNCSCI datasets of 1594 patients was used for validation and for evaluation of determinability.

\section{Derivation/validation}

In an iterative process, algorithms were primarily adjusted to process all datasets that were free of NT data (5049). Determinability algorithms were adjusted afterwards using 493 datasets of the EM-SCI database containing 1-10 NT datum/ data. Then, representative datasets were constructed for each borderline decision-rule (e.g., determinability for each output variable was analyzed for any possible input variable constellation by mixing regular scores with NT scores). In a final step, so-called "unexpected and challenging cases" were retrieved from the EM-SCI database and classified. Eventually, no differences between computer- and non-computer-derived calculations by clinical experts were found. Interested readers are encouraged to visit the interactive web application (http://ais.emsci.org) to test the described algorithms.

\section{Determinability}

The frequency of at least one and at most $10 \mathrm{NT}$ scores per dataset was $493 / 5542(8.90 \%)$. The upper boundary of $10 \mathrm{NT}$ data was chosen because it reflects an entirely NT motor examination in a lower or upper extremity. Furthermore, this rule excludes datasets graded entirely NT in PP and LT, due to diminished or altered sensation of reference facial dermatomes. Distribution of NT data was $56.11 \%$ and $43.89 \%$ for motor and sensory examinations, respectively. AIS was determinable in $412 / 493(83.6 \%)$ datasets. Figure 4 shows a more detailed 


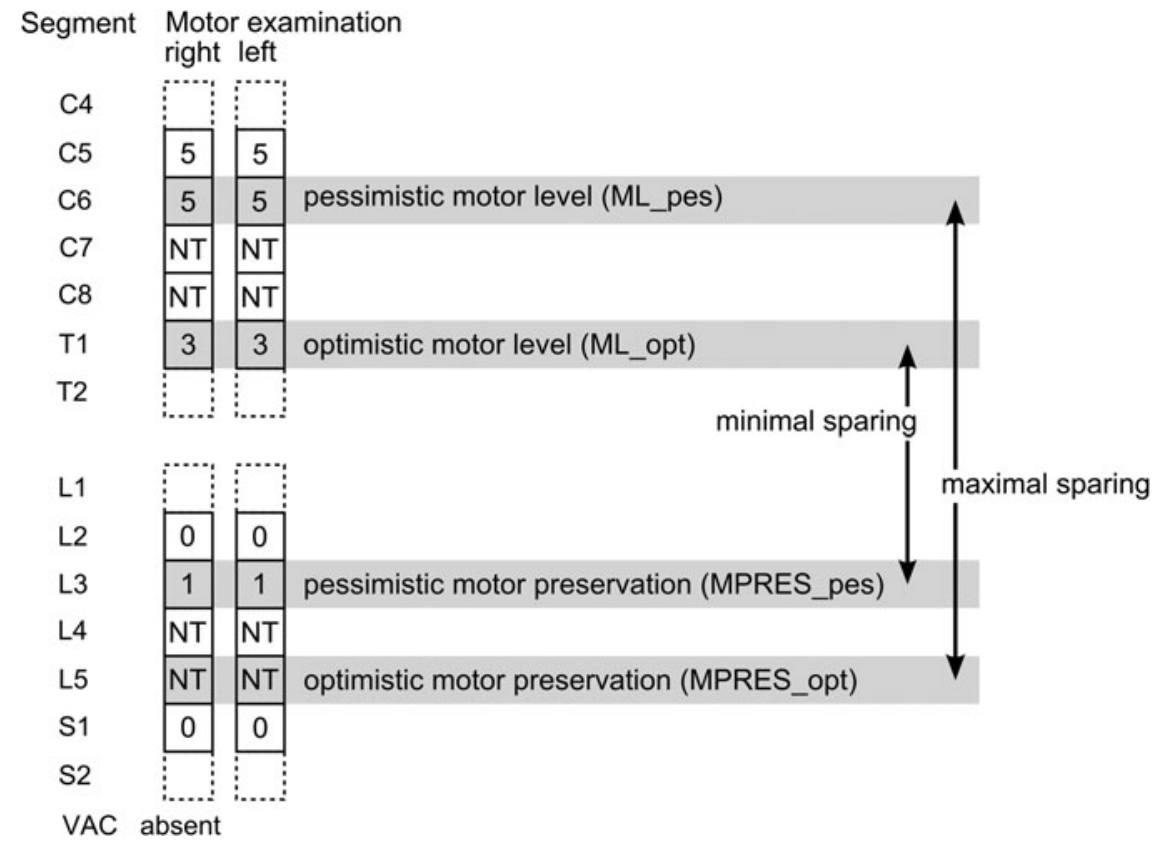

FIG. 3. Example of the motor incompleteness determination for datasets containing not testable (NT) data in the motor examination. First, minimal and maximal sparing of motor function is calculated. The decision rule for AIS B is defined by min. sparing $\leq 3$ and max. sparing $\leq 3$. Determinability is checked by the expression: min. sparing $\leq 3$ and max. sparing $>3$. The AIS is not determinable if this expression is true. For all other cases, the degree of motor incompleteness is computed (see section on key muscle counting criteria; AIS, American Spinal Injury Association Impairment Scale; VAC, voluntary anal contraction).

analysis depending on the number of NT data. In datasets with 10 NT scores, AIS determinability was still $75 \%$.

\section{Implementation}

The presented algorithms were successfully developed and are applicable for both manual and computational scaling, scoring, and classification. The source code of the computational implementation for the core library functions has a length of about 1900 lines of code. Besides the implementation in the database of the EM-SCI project, a freely available implementation can be found at http://ais.emsci.org, in the form of a simple web application. This implementation accounts for approximately 1100 lines of C source code. Classification

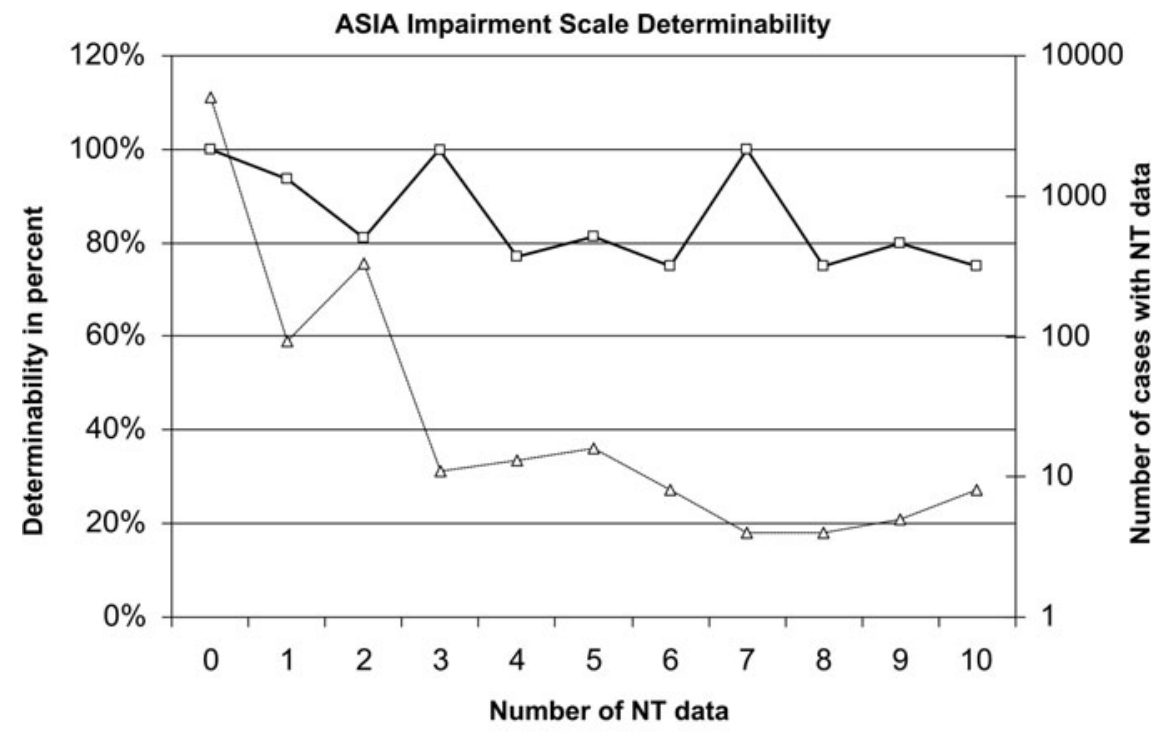

$\neg-$ AIS determinability $\rightarrow-$ Number of case with NT data

FIG. 4. Determinability of the American Spinal Injury Association Impairment Scale (AIS) depending on the number of not testable (NT) data (x-axis). Determinability is assigned to the left y-axis. The absolute number of NT data is shown in a logarithmic order on the right $\mathrm{y}$-axis. 
speed on decent hardware $\left(\right.$ Intel ${ }^{\circledR}$ Core2Quad $^{\mathrm{TM}}$ Q9400 @ 2.66 $\mathrm{GHz}, 4 \mathrm{~GB} \mathrm{RAM}$ ) is about 225,000 ISNCSCI classifications per second. In comparison, manual classification speed is estimated around 1-2 classifications per minute.

\section{Discussion}

Only recently, computer programs that allow the reliable classification of ISNCSCI source data (Wang et al., 2002) have become available (Chafetz et al., 2009; Linassi et al., 2010). The present study successfully implemented algorithms with the ability to manage the frequent problem of NT segments (up to $10 \%$ in the EM-SCI database). The employed computational algorithms were as accurate as manual classification of SCI severity scores. Determinability was excellent, even in cases with up to 10 NT segments. In contrast to manual classification, computational algorithms were much more efficient, particularly for large numbers of datasets.

\section{Algorithms}

The presented algorithm for NLI classification (the logical approach) is a successor of a previously reported algorithm (Spiess et al., 2009), which used a brute force technique to substitute all possible examination grades for NT data recursively. This algorithm is very easy to implement; however, it prolongs runtime, which depends on missing data in a quadratic to cubic order (e.g., NLI classification for datasets with 14 NT data requires several minutes, even when using decent hardware).

Proper algorithmic adjustment relies on the availability of a variety of real-world datasets. Constructed cases are not sufficient to reflect the whole spectrum of database variability. Real-world datasets contain combinations of parameter values that are often unimaginable by creators of defined standards using constructed cases.

Challenging aspects of the ISNCSCI include the use of two sensory modalities, because this leads to increased complexity in sensory level and sensory ZPP determination. Motor levels and motor ZPPs contain additional demanding aspects (i.e., not assessable segments C2-C4, T2-L1, and S2-S4-S5). A transfer function was introduced to predict motor function from sensory function, as required by the ISNCSCI. As opposed to Chafetz's (Chafetz et al., 2009) nested IF-THEN statements, this approach was chosen because it requires fewer statements and no case differentiations. This allows better readability and enhanced maintenance. In particular, the critical segments $\mathrm{C} 3$ and $\mathrm{L} 1$ are difficult to determine with nested IF-THEN statements.

The algorithms do not include optional muscle testing, as suggested by the standards to discriminate between sensory incomplete and motor incomplete lesions (American Spinal Injury Association, 2003, p. 57). The introduction of optional muscle testing into the presented algorithms is easily possible. However, this modification was not considered for the EMSCI project ((see Waring et al., 2010) for a controversial discussion and the official ASIA statement).

\section{Limitations of the definitions/ISNCSCI}

During the implementation and testing of the algorithms, several limitations of the standards have become obvious, in particular, the lack of guidance for handling NT segments in sensory levels, motor levels, ZPPs, neurological level of injury, and the ASIA Impairment Scale. The presented algorithms use a logical approach to circumvent these limitations (i.e., only when all possible NT data lead to an unambiguous result, the specified output variable is generated).

In the ISNCSCI, a clear guideline whether motor ZPPs follow sensory ZPPs or not is missing. The present article describes algorithms for both cases. The "motor follows sensory" rule leads to the question of how exactly a completely intact motor function is defined for low sacral lesions. One may think of a definition, such as, "All key muscles are graded 5 and voluntary anal contraction is present." But this definition contradicts the "motor follows sensory" rules, because the region S2-S5 is also covered by the rule. Therefore, a sensory impairment in this region implies a low sacral motor impairment. It can be argued that this type of lesion is not an SCI per se, but is a cauda equina lesion. For the purposes of a set of rules for standards covering all aspects of an SCI, this issue needs to be addressed. Another question, in the context of low sacral lesions, is how to distinguish between AIS C and AIS D below S1, where key muscles are not defined. Mathematically, this situation results in the equation $0 \geq 0$ (zero key muscles graded three or better and zero overall key muscles below NLI), using the rule that "at least half of the key muscles below the neurological level of injury must have a muscle grade greater than or equal to $3^{\prime \prime}$ (American Spinal Injury Association, 2003, p. 54). This is true for all lesions below S1 (myotomes S2-S5 are not assessable), resulting in an AIS D grade (assuming VAC is present). A clarification in the standards, whether this assumption was intended by the ASIA, would be helpful.

The difficulties arising in determining the exact sensory and motor levels at the rostral end of the spinal cord have been clarified recently (American Spinal Injury Association, 2011). Now, by definition an already impaired segment $\mathrm{C} 2$ implies a sensory/motor level of $\mathrm{C} 1$. Prior to this definition in the present study it was decided to set the level to C0-1, indicating that the lesion is rostral to $\mathrm{C} 2$. This was in particular important for the consistent determination of the NLI. Setting the NLI to C0-1 permitted the unmodified AIS algorithms to be applied to this kind of lesion. Eventually, consensus regarding this point needs to be reached.

\section{Teaching tool}

The freely available web application at http://ais.emsci.org may serve as a valuable teaching tool for all ISNCSCI examiners. It provides a simple interface to check individual classification skills by comparing them to the computational classification results.

\section{Future work}

It is planned to iteratively extend the first version of the web application for set-up of an interactive e-learning platform. For this purpose a maximum transparency of the computational decision-making process is essential to enhance the understanding of a user. As a starting point a graphical representation of the decision tree (Fig. 2) used for the classification of the individual dataset will be implemented.

We strongly believe that computer-based classification algorithms form a valuable tool for learning and quality control. Nevertheless, good manual classification skills are a 
mandatory prerequisite, together with excellent examination skills, for a qualified ISNCSCI examiner.

During the implementation of the algorithms used in this study it became obvious that the current ISNCSCI framework still leaves room for interpretation in difficult cases. These difficult cases can be identified by the analysis of real datasets from large databases (e.g., EM-SCI or Model Spinal Cord Injury System), with several computer implementations. Those datasets, in which the classifications results differ from each other, may serve as a reference for reaching a consensus among ISNCSCI experts and help to enhance the integrity of the standards.

\section{Conclusions}

Computational algorithms allow efficient and human-error-free classification of the ISNCSCI scores. Reliable assessment is clinically relevant, since these scores represent the basis of early predictions of recovery after SCI. Our computational algorithm extends the clinical applicability of computer programs by integrating NT segments, which are a frequent problem in clinical practice. With the algorithms and the computational framework, different classification rules can be compared efficiently within a short period of time, even with larger datasets.

\section{Acknowledgments}

The authors thank all SCI centers that are constantly contributing patient data to the EM-SCI study. Special thanks go to Daniel Klaus for his patience when evaluating literally thousands of ISNCSCI datasets. We are grateful to Julianne McCall, M.S., for editorial support. This work was partly funded by the International Foundation for Research in Paraplegia (IFP).

\section{Author Disclosure Statement}

No competing financial interests exist.

\section{References}

American Spinal Injury Association (ASIA). (2011). International Standards for Neurological Classification of Spinal Cord Injury revised 2001; Atlanta, GA. Reprinted 2011.

American Spinal Cord Injury Association (ASIA). (2003). Reference Manual for International Standards for Neurological Classification of Spinal Cord Injury, revised 2003. Chairman and Editor: R.J. Marino. ASIA: Chicago, IL.

Bieman, J.M., and Murdock, V. (2001). Finding code on the World Wide Web: a preliminary investigation. Proceedings First International Workshop on Source Code Analysis and Manipulation (SCAM 2001), Nov. 2001.

Chafetz, R.S., Prak, S., and Mulcahey, M.J. (2009). Computerized classification of neurologic injury based on the international standards for classification of spinal cord injury. J. Spinal Cord Med. 32, 532-537.

Chafetz, R.S., Vogel, L.C., Betz, R.R., Gaughan, J.P., and Mulcahey, M.J. (2008). International standards for neurological classification of spinal cord injury: training effect on accurate classification. J. Spinal Cord Med. 31, 538-542.

Cohen, M.E., Ditunno, J.F., Donovan, W.H., and Maynard, F.M. (1998). A test of the 1992 International Standards for Neurological and Functional Classification of Spinal Cord Injury. Spinal Cord 36, 554-560.
Curt, A., Schwab, M.E., and Dietz, V. (2004). Providing the clinical basis for new interventional therapies: refined diagnosis and assessment of recovery after spinal cord injury. Spinal Cord 42, 1-6.

Ditunno, J.F. (2010). Outcome measures: evolution in clinical trials of neurological/functional recovery in spinal cord injury. Spinal Cord 48, 674-684.

Fawcett, J.W., Curt, A., Steeves, J.D., Coleman, W.P., Tuszynski, M.H., Lammertse, D., Bartlett, P.F., Blight, A.R., Dietz, V., Ditunno, J., Dobkin, B.H., Havton, L.A., Ellaway, P.H., Fehlings, M.G., Privat, A., Grossman, R., Guest, J.D., Kleitman, N., Nakamura, M., Gaviria, M., and Short, D. (2007). Guidelines for the conduct of clinical trials for spinal cord injury as developed by the ICCP panel: spontaneous recovery after spinal cord injury and statistical power needed for therapeutic clinical trials. Spinal Cord 45, 190-205.

Furlan, J.C., Noonan, V., Singh, A., and Fehlings, M.G. (2011). Assessment of impairment in patients with acute traumatic spinal cord injury: A systematic review of the literature. J. Neurotrauma 28, 1445-1477.

Linassi, G., Shan, R.L.P., and Marino, R.J. (2010). A web-based computer program to determine the ASIA impairment classification. Spinal Cord 48, 100-104.

Marino, R.J., Barros, T., Biering-Sorensen, F., Burns, S.P., Donovan, W.H., Graves, D.E., Haak, M., Hudson, L.M., and Priebe, M.M. (2003). International standards for neurological classification of spinal cord injury. J. Spinal Cord Med. 26 Suppl. 1, 50-56.

Mulcahey, M.J., Gaughan, J., Betz, R.R., and Vogel, L.C. (2007). Rater agreement on the ISCSCI motor and sensory scores obtained before and after formal training in testing technique. J. Spinal Cord Med. 30 Suppl. 1, 146-149.

Rupp, R., Schweidler, J., Curt, A., Dietz, V., and Gerner, H.J. (2005). An electronic tool for multicenter administration, assessment and analysis of clinical trials in spinal cord injury. Biomedizinische Technik Nürnberg 1460-1461.

Spiess, M.R., Mueller, R.M., Rupp, R., Schuld, C., and van Hedel, H.J.A. (2009). Conversion in ASIA Impairment Scale during the first year after traumatic spinal cord injury. J. Neurotrauma 26, 2027-2036.

van Middendorp, J.J., Hosman, A.J., Donders, A.R., Pouw, M.H., Ditunno, J.F. Jr., Curt, A., Geurts, A.C., Van de Meent, H.; EMSCI Study Group. (2011). A clinical prediction rule for ambulation outcomes after traumatic spinal cord injury: a longitudinal cohort study. Lancet 377, 1004-1010.

Wang, D., Taylor, B., Gardner, B., and Frankel, H. (2002). A computerized program for neurological classification of spinal cord injury according to the ASIA/IScoS international standards. Annual meeting of the International Spinal Cord Society (ISCOS), 2002, Vancouver, Canada.

Waring, W.P. 3rd, Biering-Sorensen, F., Burns, S., Donovan, W., Graves, D., Jha, A., Jones, L., Kirshblum, S., Marino, R., Mulcahey, M.J., Reeves, R., Scelza, W.M., Schmidt-Read, M., and Stein, A. (2010). 2009 review and revisions of the international standards for the neurological classification of spinal cord injury. J. Spinal Cord Med. 33, 346-352.

Yuan, Y., and Shaw, M.J. (1995). Induction of fuzzy decision trees. Fuzzy Sets and System 69, 125-139.

Address correspondence to: Christian Schuld, Dipl.-Inform. Med. (equivalent to M.Sc.) Heidelberg University Hospital Spinal Cord Injury Center Experimental Neurorehabilitation Schlierbacher Landstraße 200a, 69118 Heidelberg, Germany E-mail: Christian.Schuld@med.uni-heidelberg.de 
